CERN-EP/99-167

26 October 1999

\title{
CHARACTERISATION OF A SINGLE PHOTON COUNTING PIXEL SYSTEM FOR IMAGING OF LOW-CONTRAST OBJECTS
}

\author{
B. Mikulec \\ CERN, Geneva, Switzerland and Universität Wien, Vienna, Austria \\ M. Campbell \\ CERN, Geneva, Switzerland \\ G. Dipasquale \\ INFN Pisa, Pisa, Italy \\ C. Schwarz \\ Universität Freiburg, Freiburg, Germany \\ J. Watt \\ University of Glasgow, Glasgow, Scotland
}

\begin{abstract}
In the framework of the Medipix ${ }^{1}$ collaboration the PCC, a single photon counting pixel chip, has been developed [1] with the aim of improving the contrast resolution in medical imaging applications. The PCC consists of a matrix of $64 \times 64$ square pixels with $170 \mu \mathrm{m}$ side length, each pixel comprising a 15 bit counter and a pulse height discriminator. The chip has been bump bonded to equally segmented $200 \mu \mathrm{m}$ thick SI-LEC GaAs detectors showing a very high absorption energy for X-rays used in diagnostics.

An absolute calibration of the system with a radioactive source and a synchrotron beam are described resulting in the value of the test input capacitance of $\sim 24.7 \mathrm{fF}$. Using this value a full characterisation of the system from electrical measurements is presented. The entire system can reach a minimum threshold of $\sim 2100 \mathrm{e}^{-}$with $\sim 250 \mathrm{e}^{-}$rms noise. One of the characteristics of the PCC is the possibility to adjust the thresholds of all pixels on a pixel-bypixel basis with 3 bit precision. The threshold distribution after adjustment is $\sim 120 \mathrm{e}^{-} \mathrm{rms}$. The spatial resolution of the system has been measured to be $3.6 \mathrm{lp} / \mathrm{mm}$. A comparison of a tooth image taken with the PCC and with a screen-CCD system demonstrates its imaging capabilities.
\end{abstract}

(Submitted to Nuclear Instruments and Methods in Physics Research A)

\footnotetext{
${ }^{1}$ The Medipix collaboration is formed by CERN, the University of Freiburg, University of Glasgow and the Universities and INFN of Pisa, Napoli and Sassari.
} 


\section{INTRODUCTION}

Most medical imaging systems still consist of film and screen-film combinations, but digital imaging systems are gradually replacing them. Digital systems offer immediate results on-screen (no film development!), easy data storage and data transmission for consulting purposes as well as the possibility of offline image processing. Digital X-ray imaging has two possible approaches: charge integration and photon counting. Most of the existing systems are based on charge integrating devices, e.g. CCDs coupled to scintillators. In photon counting mode a threshold in energy is set in each pixel. Therefore the main advantage of photon counting is that noise is suppressed and detector leakage current and electronics mismatch can be compensated for which results in a large and linear dynamic range. That leads to the expectation that photon counting should improve the image quality of lowcontrast objects.

This principle has been taken up by the Medipix collaboration resulting in the PCC, a single photon counting pixel chip. The chip has an active area of $\sim 1.2 \mathrm{~cm}^{2}$ segmented into $64 \times 64$ pixels of size $170 \times 170 \mu^{2}$. Each pixel comprises a preamplifier with leakage current compensation circuitry, a discriminator and a 15 bit counter. Besides a threshold setting for the whole chip the thresholds of individual pixels can be tuned with a 3 bit threshold adjust. Each pixel has one mask and one test bit. For electrical measurements a test-pulse is injected via a test-input capacitance. The value of this capacitance being very small (design value: $20 \mathrm{fF}$ ) can vary significantly from one chip processing run to the next. Therefore it is necessary to calibrate the capacitance in order to be able to transform electrical measurements $[\mathrm{mV}]$ into absolute charge values [e]. An absolute calibration with a radioactive source has been performed and is explained in section 2 .

The technique of hybrid assemblies involves connecting the electronics chip together with the sensor through flip-chip processing. This leaves free the choice of the detector material $^{2}$. The PCC has been bump-bonded to a GaAs sensor and section 3 is dedicated to detector measurements.

In section 4 a system characterisation with electrical measurements is presented and results are given in absolute values.

Finally some images are shown in section 5.

\footnotetext{
${ }^{2}$ In the case of the PCC not all materials can be used as sensors because the electronics are conceived to collect only holes. Materials with a very short hole lifetime (e.g. CdTe) therefore cannot be considered.
} 


\section{ABSOLUTE CALIBRATION OF THE TEST-INPUT CAPACITANCE}

The calibration procedure must establish a relation between the electrical signal measured with the system and a well defined value of collected charge. It is common practice to use radioactive gamma sources for this purpose. As the PCC was originally designed for mammographic applications a ${ }^{109} \mathrm{Cd}$ source is a very good candidate being a pure gamma source emitting X-rays with energies lying close to the energies used for mammography. The two $\mathrm{K}_{\alpha}$ lines (having the highest relative intensities) lie closely around $22 \mathrm{keV}$ and the two $\mathrm{K}_{\beta}$ lines around $25 \mathrm{keV}$. At $88 \mathrm{keV}$ there is another gamma emission line with low relative intensity, which will give a small background to the measurement described below.

The principle of the measurement is to start from a discriminator threshold well below the gamma lines and to move the threshold up in very fine steps. As soon as one passes the gamma energy there will be a drop in counts, or a peaked distribution after differentiation. In the case of a ${ }^{109} \mathrm{Cd}$ source the ideal situation would be if the detector system could separate at least the two peaks at 22 and $25 \mathrm{keV}$. This was possible in the case of the LHC1/Omega3 chip [2], the predecessor of the PCC from the domain of high-energy physics, when it was bump-bonded to a silicon detector [3]. For the PCC the overall noise was too large and prevented separation of the two peaks. Nevertheless the threshold at which the counts drop almost to zero (except for the few counts originating from the $88 \mathrm{keV}$ line) can be clearly determined and with an error caused by the noise corresponds to the highest gamma energy $(\sim 25.6 \mathrm{keV})$. A photon with this energy deposits about $6000 \mathrm{e}^{-}$in GaAs [4]. It is important to mention that the whole analysis has been performed on a pixel-by-pixel basis. The discriminator thresholds in each individual pixel marking the end-points of the spectrum are then compared with the electrically measured threshold in $\mathrm{mV}$ for that pixel at the specific threshold setting. Assuming a charge collection efficiency (CCE) of 89\% measured for our detector material [5] it is now easy to calculate the value for the test-input capacitance as $\sim 23.4 \mathrm{fF}$ (see fig. 1). As the system noise always broadens the original gamma line the value of $23.4 \mathrm{fF}$ corresponds to a low limit for the capacitance. In order to correct for this broadening effect we subtracted $250 \mathrm{e}$, corresponding to one sigma of our system noise (see section 4), which gives the final result of $\sim 24.7 \mathrm{fF}$ for the test-input capacitance. The location of the square in fig. 2 corresponds in $\mathrm{x}$ to the average threshold voltage setting of the chip where the end-points were found and in y to the average pixel thresholds measured with test pulses. The curve itself shows the average value of the thresholds of all 4096 pixels as a function of threshold setting, the error bars being the rms of the threshold distribution. It can be seen that the discriminator has only a limited linear range of about $3000 \mathrm{e}^{-}$with the $\mathrm{Cd}$ calibration point lying already at the upper edge of the linear region. The rms of the threshold distribution is smallest at the threshold setting where the threshold adjustment mask (see section 4$)$ has been created $(1.35 \mathrm{~V})$. The calibration point lies slightly under the curve as a selection of 230 pixels was taken into account for the analysis. The selected pixels were situated around the centre of the source for statistics reasons and showed a rather low threshold which made it easier to follow the entire spectrum and determine correctly its endpoint. 
The calibration result was confirmed by analysing data taken at the Daresbury synchrotron [6]. The same method as described was used, but this time with a (monoenergetic) $14.27 \mathrm{keV}$ synchrotron beam. There the average threshold setting corresponding to the end-points was found at $\sim 1.25 \mathrm{~V}$ and a respective threshold of $\sim 3000 \mathrm{e}^{-}$ being in good agreement with the ${ }^{109} \mathrm{Cd}$ calibration. Nevertheless it should be mentioned that this calibration was performed with one assembly only; the value for the test-input capacitance might change slightly for others. Due to the fact that the ${ }^{109} \mathrm{Cd}$ peaks could not be resolved it should be considered as a good approximation. Moreover the value of $89 \%$ for the $\mathrm{CCE}$ has been determined with a shaping time of $1 \mu \mathrm{s}$. The PCC has a shaping time of $150 \mathrm{~ns}$ which means that part of the signal could be lost. In that case the value for the test-input capacitance would represent an overestimation and all the absolute values in electrons given in the following sections should be considered as worst case values.

A more precise calibration value may be obtained using silicon detectors bump-bonded to the PCC.

\section{SI GaAs DETECTOR PERFORMANCE}

As sensor material semi-insulating (SI) GaAs was chosen due to its high absorption efficiency for X-rays up to about $30 \mathrm{keV}$ (see fig. 3) being $\sim 100 \%$ up to $20 \mathrm{keV}$. The raw material originates from Sumitomo and has been processed by Alenia [8]. One of the problems of SI GaAs is that it usually reaches breakdown before reaching full CCE. Alenia developed a new backside contact (non-alloyed ohmic contact NAOC [9]) making it possible to bias $200 \mu \mathrm{m}$ thick detectors up to voltage regions around $600 \mathrm{~V}$.

In order to evaluate the detector bias voltage at which the active layer extends to the detector thickness $(200 \mu \mathrm{m})$ we illuminated the detector from the backside with a pure alpha source, ${ }^{210} \mathrm{Po}$. The energy of the alpha emission of ${ }^{210} \mathrm{Po}$ is $\sim 5.4 \mathrm{MeV}$ leading to a penetration depth of only $\sim 20 \mu \mathrm{m}$ [10]. As can be seen from fig. 4 the detector volume is fully active above $\sim 260 \mathrm{~V}$ where a plateau in counting rate is reached. There is a region of a steep increase in counts from $\sim 170-260 \mathrm{~V}$ between where the electric field reaches the charge deposition region and until it reaches the backside. The error bars visualising the rms of the distribution of counts are also very large for the transition bias conditions getting almost constant in the plateau. The small excess of counts at $260 \mathrm{~V}$ might be due to charge injection from the backside contact when reached by the electric field.

The transition region to fully active detector volume is also visible as a small bump between $\sim 190-280 \mathrm{~V}$ in the I-V measurement of the detector as shown in fig. 5. After this region the leakage current increases steadily, but without immediate breakdown of the diode. Measurements have shown that also the CCE increases slowly in this region [9,11]. Making a compromise between a leakage current that is not too high and a maximum detection efficiency of the detector we made most of our measurements at a detector bias of $340 \mathrm{~V}$. 
For the distinction of low-contrast objects it is very important that all pixels in the chip matrix have a homogeneous threshold. To achieve this the chip has 3-bit threshold tuning in each pixel. The left-most distribution in fig. 6 shows the threshold distribution of one assembly without this threshold optimisation, its rms being $\sim 500 \mathrm{e}$. The distribution on the right side is the one for maximum adjustment. After optimisation one can obtain $\sim 120 \mathrm{e}$ rms (measured with $340 \mathrm{~V}$ detector bias). This value has to be compared with the system noise (fig. 7), which lies around $\sim 250 \mathrm{e}^{-}$measured as described in [12]. Therefore the system performance is noise limited at present.

The adjusted distribution can afterwards be shifted down to lower thresholds reducing the global threshold setting. The minimum threshold we could achieve with our assemblies without bias was $2100 \mathrm{e}^{-}$(fig. 8a)), with bias $2400 \mathrm{e}^{-}$(fig. 8b)).

\section{IMAGING}

The spatial resolution was determined with a line-pair mask to be $3.6 \mathrm{lp} / \mathrm{mm}^{3}$ (see fig. 9). The part on the bottom of the image corresponds to an area of bad bump bonding.

A first comparison between the performance of an integrating system and the PCC photon counting system has been carried out. A tooth has been imaged with a conventional X-ray tube (Trophy type 708, long cone, $8 \mathrm{~mA}, 70 \mathrm{kV}, 0.2 \mathrm{~s}$ acquisition time) and a CCD detection system. Fig. 10a) shows the scanned image with a screw getting thinner the deeper it sits inside the tooth. The detection system should be able to image the screw until its end. As a comparison we made 2 images (one of the top and the other of the bottom part of the tooth due to the relatively small detection area) with the PCC (fig. 10b)) using ${ }^{109} \mathrm{Cd}$ as X-ray source. The spatial resolution is clearly poorer than that of the CCD system because of the pixel size ${ }^{4}$, but nevertheless the screw can be clearly distinguished from the surrounding tooth material. Moreover the differences in tooth density are much more clearly visible. In reference [13] the exposure $[\mu \mathrm{Gy}]$ as a function of exposure time for the above mentioned setup used to obtain image 10a) has been reported (tube A in ref. [13]). The acquisition time of $0.2 \mathrm{~s}$ yields therefore an exposure of $\sim 980 \mu \mathrm{Gy}$. A calculation of the directional dose equivalent, $\mathrm{H}^{\prime}(0.07)$, using the $\Gamma$-factor ${ }^{5}$ for ${ }^{109} \mathrm{Cd}[14]$ yields a value of $\sim 33 \mu \mathrm{Sv}$. With a correction factor which is very small for the ${ }^{109} \mathrm{Cd}$ energies [15] the exposure for fig. 10b) could be determined to be $\sim 34 \mu \mathrm{Gy}$ lying almost 30 times below the dose for the image with the CCD system.

\footnotetext{
${ }^{3}$ Corresponds to a resolution of $\sim 278 \mu \mathrm{m}$.

${ }^{4}$ A successor of the PCC is in its final design state with much reduced pixel size.

${ }^{5}$ Corresponds to the parameter $\mathrm{h}_{0.07}=5(\mathrm{mSv} / \mathrm{h}) / \mathrm{GBq}$ at $10 \mathrm{~cm}$ distance in ref. [14].
} 


\section{CONCLUSIONS}

In this paper we have presented the calibration of the PCC. We measured a value for the test-input capacitance of $\sim 24.7 \mathrm{fF}$ for one assembly assuming a CCE of $89 \%$ with a ${ }^{109} \mathrm{Cd}$ source, which was confirmed by a measurement using synchrotron radiation. Measurements have been carried out characterising the SI GaAs detector as well as the whole PCC system. In the last section images were presented that offer also the possibility of comparing the imaging potential of the PCC with a CCD system.

We are planning to carry out in the near future measurements with dedicated phantoms to make a detailed quantitative comparison of contrast and resolution. It is also foreseen to test the PCC bump bonded to silicon detectors to be able to separate the effects of the detector from electronics performance.

\section{Acknowledgements}

The authors would like to thank Iztok Ropotar for discussions especially concerning the chip calibration. Prof. van der Stelt helped with his expertise in imaging quality assessment. Many thanks also to Dr. Deluzurieux who provided an adult tooth for tests and the image of the same (fig. 10a)). Dr. T. Otto from the radiation protection group at CERN and Dr. F. Glasser from CEA-LETI Grenoble provided invaluable help with the dose comparison. 


\section{References}

[1] M. Campbell, E.H.M. Heijne, G. Meddeler, E. Pernigotti, W. Snoeys, Readout for a 64 x 64 Pixel Matrix with 15-bit Single Photon Counting, IEEE Trans.Nucl.Sci. 45 (3), 751, June 1998

[2] E.H.M. Heijne et al., LHC1: A Semiconductor Pixel Detector Readout Chip with Internal, Tunable Delay Providing a Binary Pattern of Selected Events, NIM A 383 (1996) 55

[3] I. Ropotar, An Investigation of Silicon Pixel Tracking Detectors and their Application in a Prototype Vertex Telescope in the CERN NA50 Heavy-Ion Experiment, doctoral thesis under preparation, University of Wuppertal, WUB-DIS 99-20

[4] J.E. Eberhardt, R.D. Ryan, A.J. Tavendale, Evaluation of Epitaxial n-GaAs for Nuclear Radiation Detection, NIM 94 (1971) 463

[5] R. Amendolia et al., Charge Collection Properties of GaAs Detectors for Digital Radiography, Physica Medica Vol. XIV, Suppl. 2, Sept. 1998, 17

[6] J. Watt et al., Applications of Pixellated GaAs X-ray Detectors in a Synchrotron Radiation Beam, proceedings of the $1^{\text {st }}$ International Workshop on Radiation Imaging Detectors, Sundsvall, Sweden, June13-17, 1999, to be published in NIM A

[7] J.H. Hubbell and S.M. Seltzer, NIST Physical Reference Data; http://physics.nist.gov/PhysRefData/XrayMassCoef/cover.html

[8] Alenia S.p.A., Via Tiburtina km 12.4, I-00131 Roma, Italy

[9] M. Alietti et al., Performance of a New Ohmic Contact for GaAs Particle Detectors, NIM A 362 (1995) 344

[10] D.S. McGregor et al., Present Status of Undoped Semi-insulating LEC bulk GaAs as a Radiation Spectrometer, NIM A 343 (1994) 527

[11] F. Nava et al., Improved Performance of GaAs Radiation Detectors with Low Temperature Ohmic Contacts, IEEE Trans. Nucl. Sci. 44 (3) (1997) 943

[12] F. Anghinolfi et al., A 1006 Element Hybrid Silicon Pixel Detector with Strobed Binary Output, IEEE Trans. Nucl. Sci. 39 (4) (1992) 654

[13] F. Glasser et al., Preliminary Characterization of a New Hybrid CdTe Structure for Xray Dental Imaging, SPIE Proceedings, Medical Imaging 1995, Physics of Medical Imaging vol. 2432 (1995) 442

[14] Radiological Protection Ordinance, The Swiss Federal Council, Annex 3, 22 June 1994

[15] W.G. Alberts et al., Neue Dosis-Meßgrößen im Strahlenschutz, Physikalisch-Technische Bundesanstalt Braunschweig, PTB-Dos-23, Juli 1994 


\section{Figure captions}

Fig. 1 Values for the test-input capacitance evaluated for 230 pixels using the end-points of the ${ }^{109} \mathrm{Cd}$ spectrum and assuming a CCE of $89 \%$. The resulting mean value is a low estimate for the capacitance as the system noise still has to be subtracted.

Fig. 2 The square shows the average threshold voltage setting of the chip which corresponds to a threshold of $25.6 \mathrm{keV}$ in GaAs assuming a CCE of $89 \%$. It was calculated with the value of $24.7 \mathrm{fF}$ for the test-input capacitance and for 230 pixels. The curve corresponds to the average threshold in electrons of the whole chip as a function of the global threshold voltage setting, the error bars being the rms of the threshold distribution.

Fig. 3 Absorption efficiency for GaAs (200 and $300 \mu \mathrm{m}$ thick), Si (300 $\mu \mathrm{m}$ and $1 \mathrm{~mm}$ ) and $\mathrm{CdTe}(300 \mu \mathrm{m})$ as a function of the photon energy ranging from $5 \mathrm{keV}$ to $100 \mathrm{keV}$ (data from [7]).

Fig. 4 Illumination of one $200 \mu \mathrm{m}$ thick GaAs detector with a ${ }^{210} \mathrm{Po}$ alpha source. Up to a detector bias of $\sim 170 \mathrm{~V}$ there are practically no counts. Going higher with the bias follows a transition region corresponding to the electric field reaching the charge deposition region and then the backplane. A plateau of counts is reached above $\sim 260 \mathrm{~V}$.

Fig. 5 Leakage current as a function of detector bias. The transition region of fig. 4 can also be observed as a small increase in leakage current. Above full activation of the detector volume the leakage current increases steadily.

Fig. 6 Principle of the threshold adjustment: The distribution on the left side is the original threshold distribution without adjustment, in the one on the right side all pixels are maximally adjusted. With 3 bits for the tuning per pixel a narrow distribution like the one in the centre can be achieved.

Fig. 7 The system noise is of the order of $250 \mathrm{e}$.

Fig. 8 a) Minimum threshold above noise without detector bias and b) with $320 \mathrm{~V}$ detector bias.

Fig. 9 Image with a line-pair mask. $3.6 \mathrm{lp} / \mathrm{mm}$ (top) can be resolved.

Fig. 10 Image of a human tooth with a fine screw inside using a conventional dental $\mathrm{X}$ ray tube and a $\mathrm{CCD}$ detection system (a)). Image b) has been taken with a ${ }^{109} \mathrm{Cd}$ source and the PCC system and is composed of 2 images. Differences in density are clearly visible. 


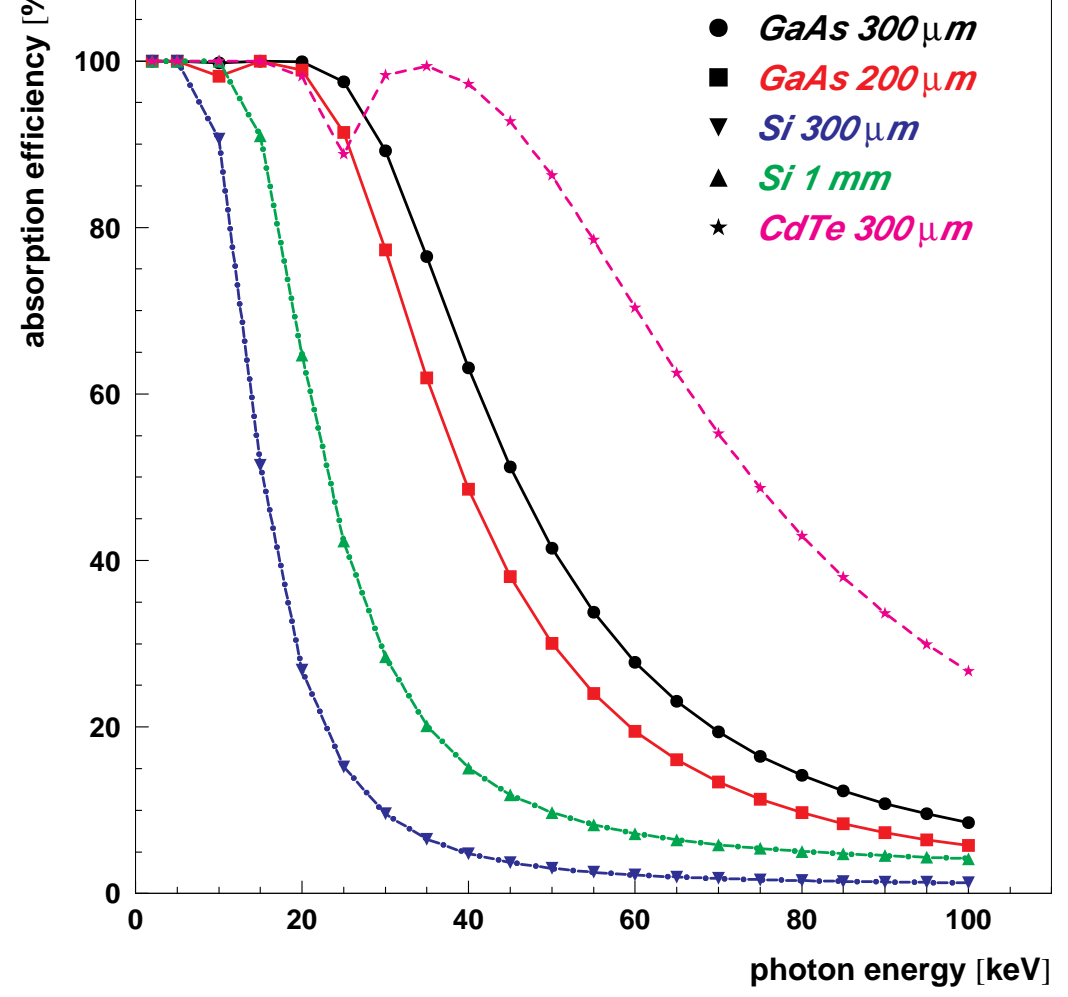

Figure 3

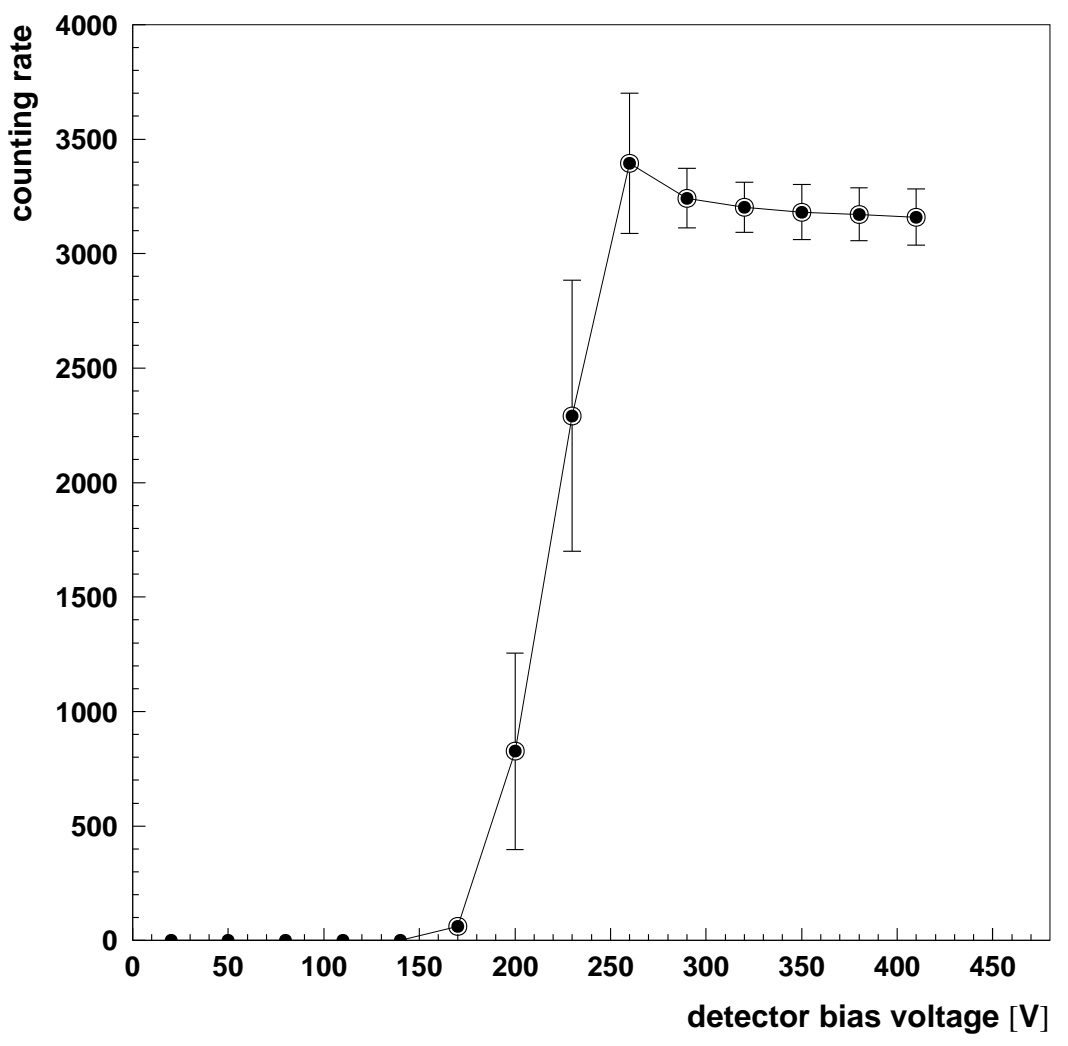

Figure 4 


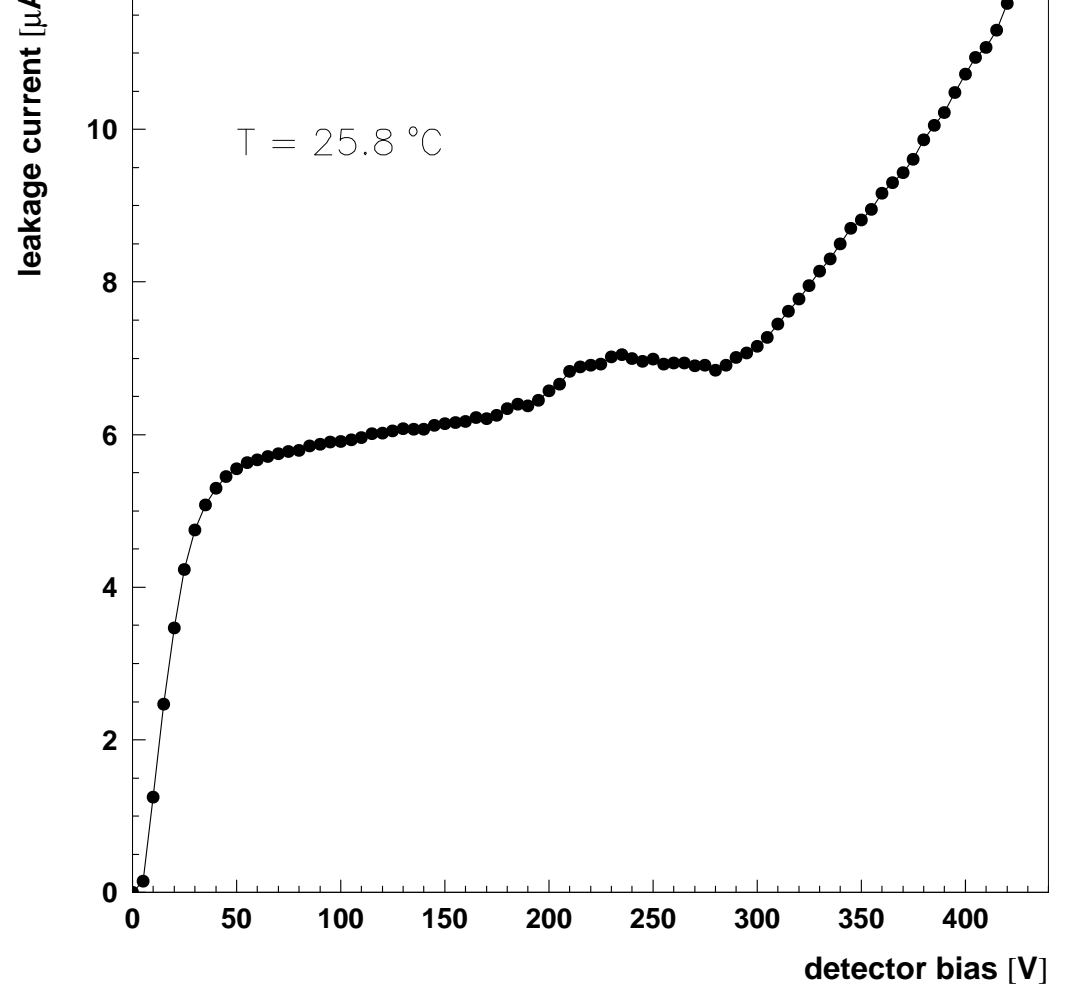

Figure 5

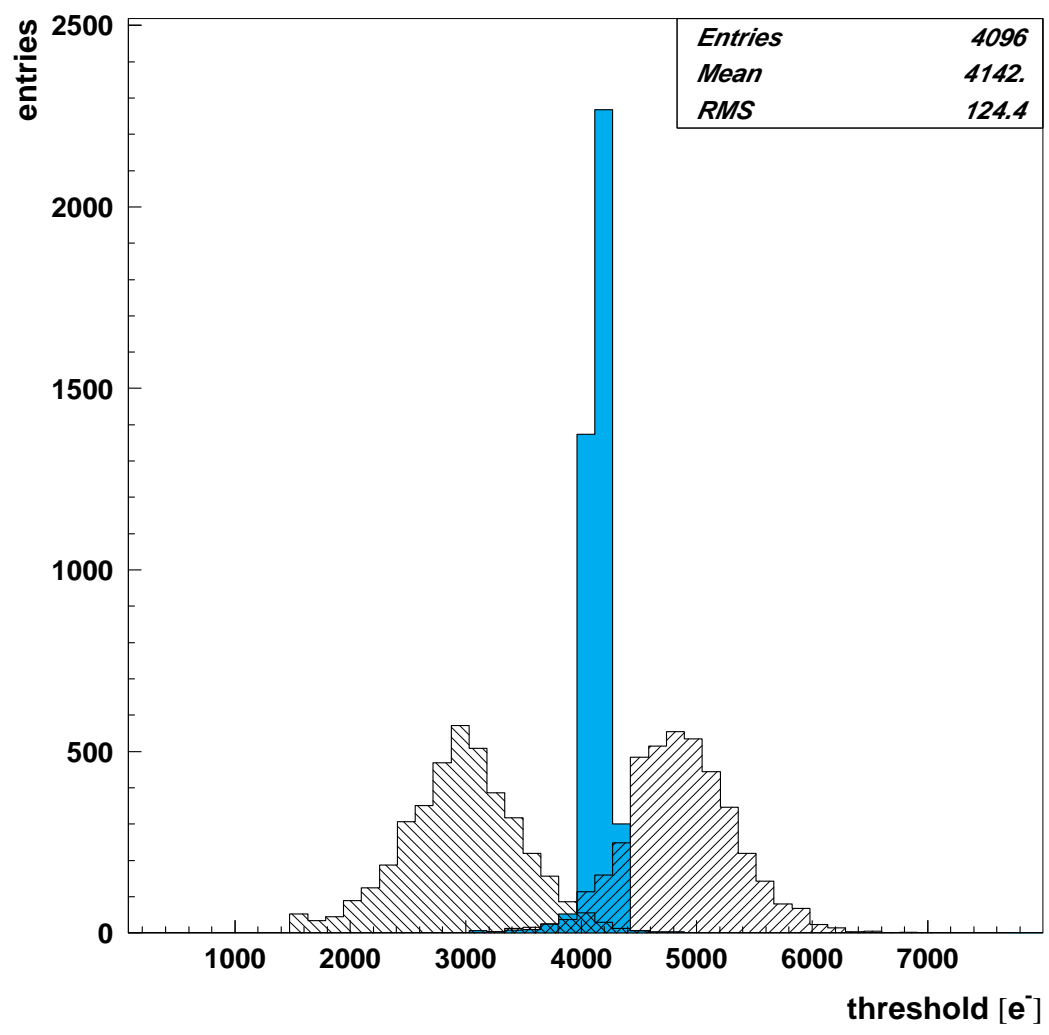

Figure 6 


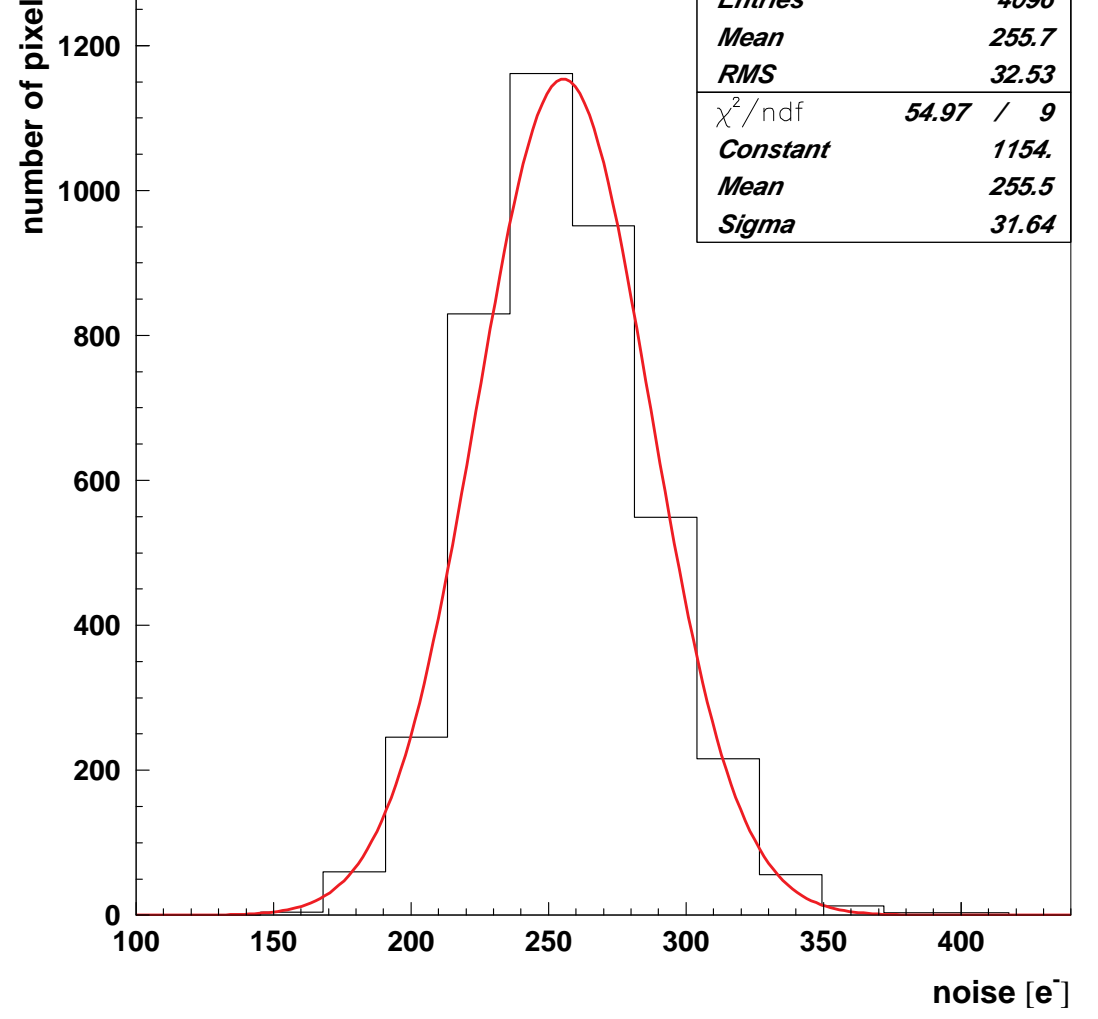

Figure 7

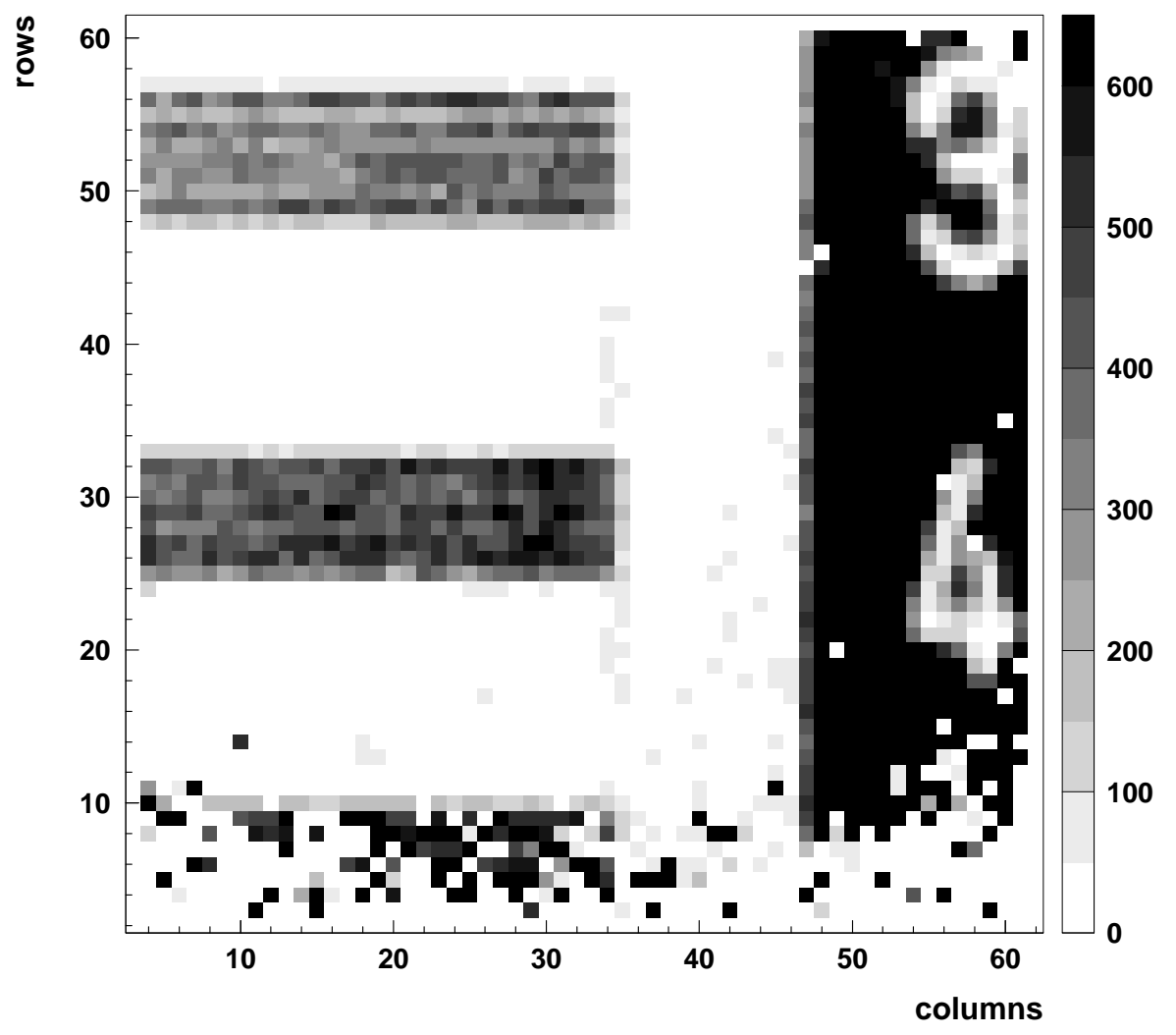

Figure 9 


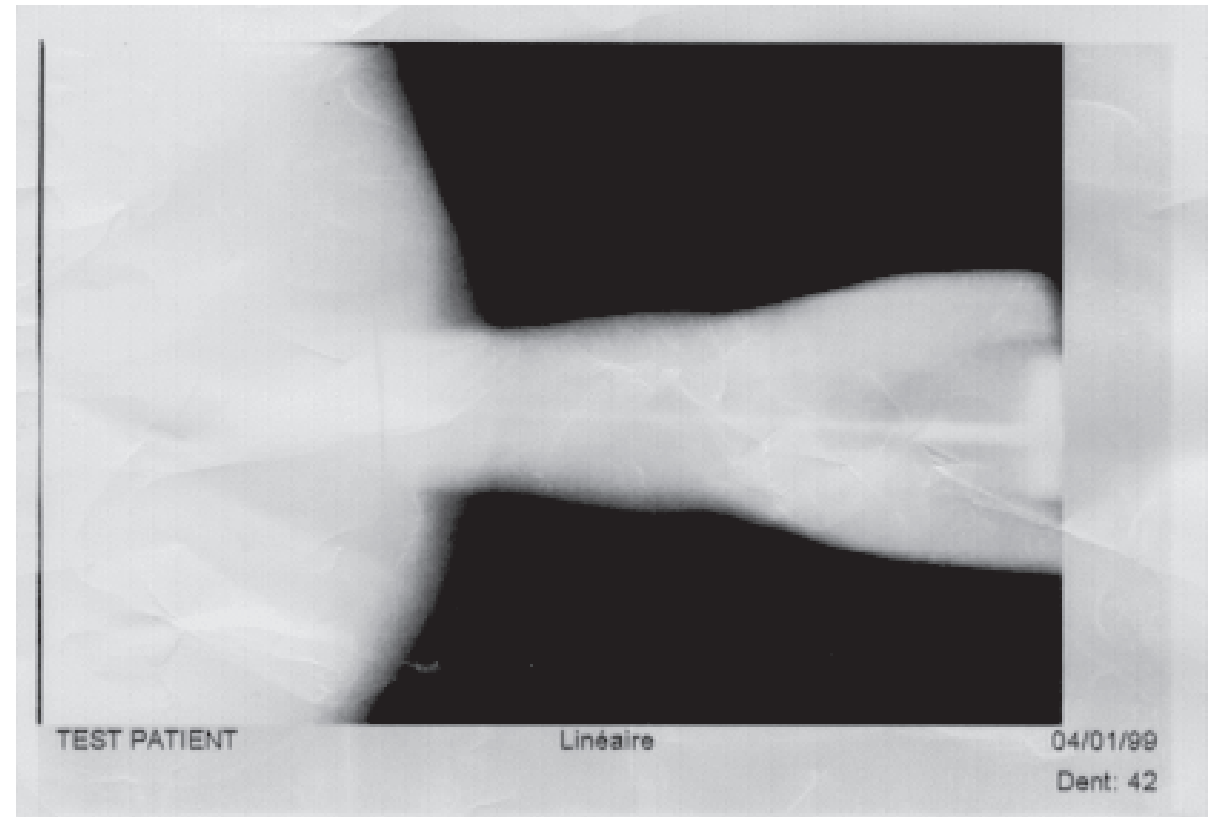

Figure 10a)

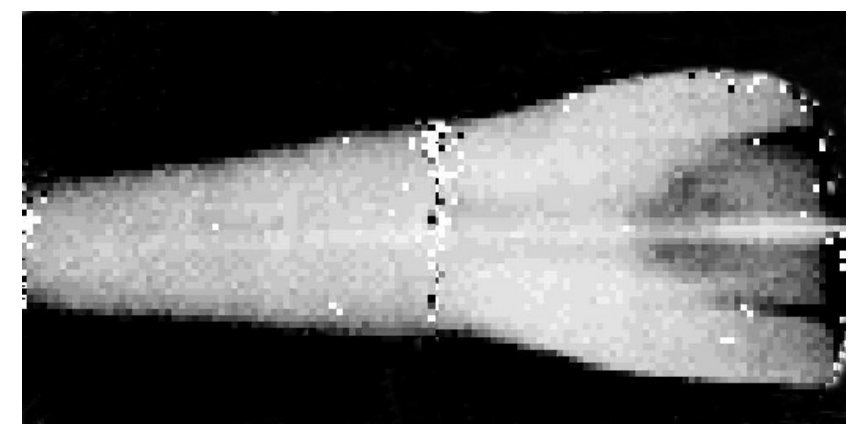

Figure 10b) 